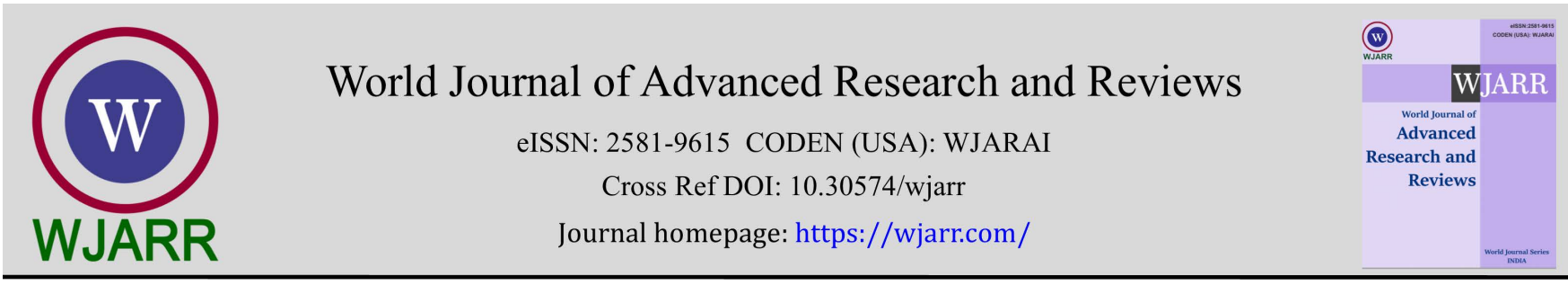

(CASE REPORT)

Check for updates

\title{
Retained surgical sponge presenting as a case of intestinal obstruction
}

\author{
Chiemelu Dickson Emegoakor *, Henry Chukwuka Nzeako, Kenneth Oluchukwu Ugwuanyi, Celestine \\ Ifeanacho Okafor and Arinze Chukwuma Ijezie
}

Department of Surgery, Nnamdi Azikiwe University Teaching Hospital Nnewi, Nigeria.

World Journal of Advanced Research and Reviews, 2021, 10(03), 412-416

Publication history: Received on 01 May 2021; revised on 25 June 2021; accepted on 27 June 2021

Article DOI: https://doi.org/10.30574/wjarr.2021.10.3.0267

\begin{abstract}
Retained surgical sponge or gossypiboma is a term used to describe a retained swab in the body after operation. There are different surgical materials that can be left in the abdomen during operation such as sponge, artery forceps, scissors, and pieces of broken instrument. Mop is the most commonly retained foreign body. Inadvertent retention of foreign body in the abdomen often requires another operation, increasing the morbidity and mortality in these patients. Despite the complications associated with this condition, they are rarely published because of medicolegal implications.
\end{abstract}

We report a case of 28-year-old woman who presented on account of 9 months history of colicky abdominal pain, abdominal distention and mass following open myomectomy in a private hospital.

Keywords: Gossypiboma; Intestinal obstruction; Laparotomy sponge; Intraluminal migration

\section{Introduction}

Retained surgical sponge otherwise called gossypiboma, textiloma or gauzoma is an uncommon but fatal complication of human surgery. It is one of the most frequent but avoidable medical errors that occur in surgical practice [1]. Gossypiboma, a term derived from the Latin "gossypium" meaning cotton and the Swahilli "boma" meaning place of concealment refers to a mass of cotton matrix retained in the body part after an operation, usually caused by abdominal surgeries. The first reported case was by Wilson in 1884 [1]. while the first malpractice suit related to gossypiboma was in 1933 [2]. Since then, the incidence of gossypiboma tend to fluctuate and difficult to estimate due to under-reporting. Although the actual incidence of this condition is not known due to medicolegal reason and many patients remain asymptomatic, it has been reported to be 1 in 1000-1500 abdominal surgeries [3].

In most cases, the gauze or mop is left in the abdomen of the patient for months to years [4]. Some reports have also demonstrated retained sponge in other body cavities such as thoracic cavity, pericardial space and paraspinal space [5]. There is usually a granulomatous reaction around the foreign body which can produce exudates with formation of abscess or a fibrotic mass. ${ }^{(5)}$ Intraluminal migration is relatively rare, leading to intestinal obstruction. The patient may initially be asymptomatic, but may progressively become symptomatic. In most cases, diagnosis is made by imaging techniques such as abdominal ultrasound scan, X-ray, CT scan or MRI. Inadvertent retention of a foreign body in the abdomen often requires another operation. This increases the patient's morbidity and mortality.

We report a case of retained mop in the abdomen following laparotomy for open myomectomy.

\footnotetext{
${ }^{*}$ Corresponding author: Chiemelu Dickson Emegoakor

Department of Surgery, Nnamdi Azikiwe University Teaching Hospiatal Nnewi, Nigeria.

Copyright $(2021$ Author(s) retain the copyright of this article. This article is published under the terms of the Creative Commons Attribution Liscense 4.0.
} 


\section{Case Report}

\subsection{Case Summary}

N.M, a 28-year-old house wife who presented with abdominal pain of 9 months duration.

Pain was noticed a day after open myomectomy. The surgery was done in a private hospital by a General practitioner in the day time. Pain was located in the left lower quadrant of abdomen, of sudden onset, colicky, last for 2 minutes with pain free interval of 15 minutes, radiates to other quadrants of the abdomen. Pain has been increasing in intensity and is worsened by eating. There was associated abdominal distension but she was still passing faeces and flatus.

There was a history of nausea, easy satiety but no vomiting. She had reduction in bowel habit from once daily to once in 2-3 days with occasional diarrhea episodes. No tenesmus, haematochezia or, nor anal protrusion. For this problem patient has taken analgesic but with no improvement. She visited the hospital where she had 2 previous laparotomies and a $3^{\text {rd }}$ laparotomy was suggested hence, she decided to present to Nnamdi Azikiwe University Teaching Hospital for proper evaluation and management. Patient had laparotomy 2 years ago at a peripheral hospital for a ruptured ectopic pregnancy surgery lasted for 3-4 hours done during day time. She is neither hypertensive nor diabetic. She has been married for 3 years but has never conceived.

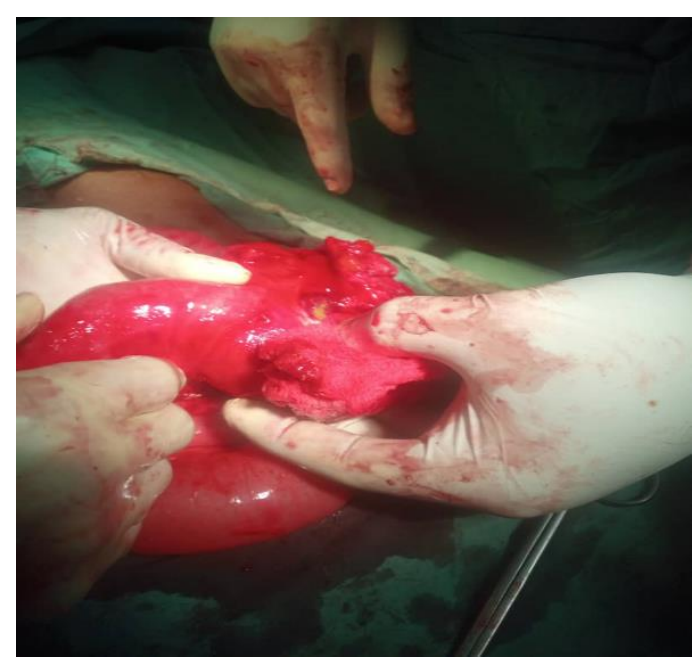

Figure 1 Mid ileal mass

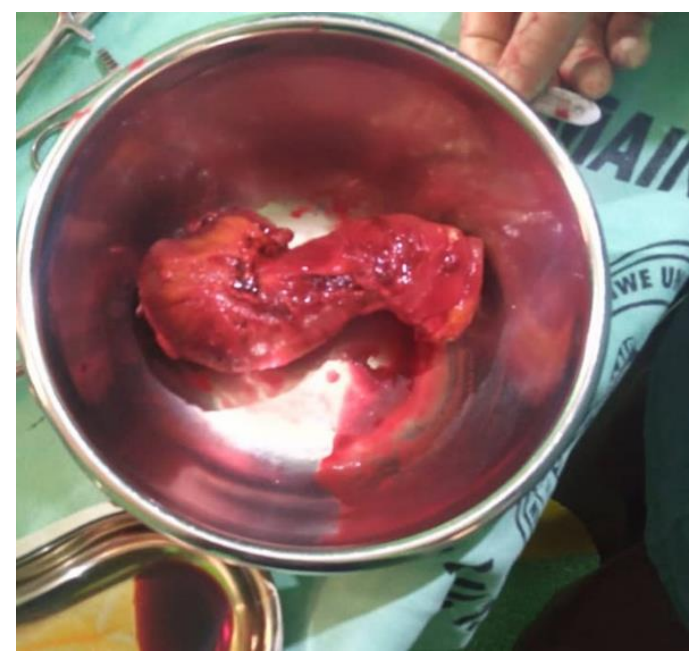

Figure 2 Resected ileum containing the intraluminal sponge

Examination revealed a young woman in obvious painful distress afebrile, not pale, anicteric, acyanosed, not dehydrated. Vital signs on presentation were stable. Chest and other regions examined revealed no abnormality. 
Abdomen was distended, moved with respiration with visible peristalsis. There was Pfannenstiel incision scar that healed with primary intention. No tenderness. There was a Mass on left iliac fossa $10 \times 8 \mathrm{~cm}$, not tender, no differential warmth firm, smooth surface, ill-defined margins not attached to the skin deep to the anterior abdominal wall. Liver spleen and 2 kidneys were not palpably enlarged. Bowel sound was hyperactive.

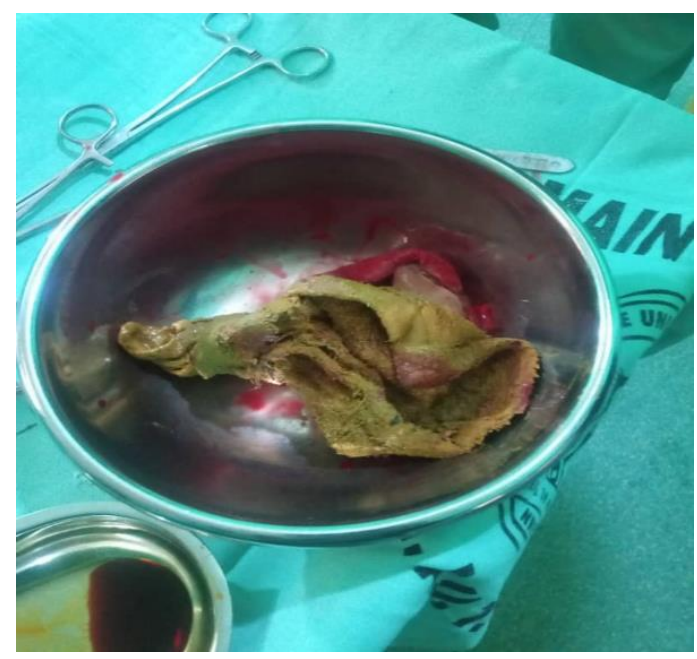

Figure 3 The sponge soaked with liquid feaces

Vaginal Examination showed No significant vaginal discharge. Cervix was nulliparous and healthy looking bilateral adnexal tenderness worse on the left. Pouch of Douglas was empty.

Rectal examination was not remarkable.

Plain Abdominal X-Ray done revealed multiple air-fluid level on erect view and dilated loops of small and large bowel on supine view. Abdominal ultrasound scan revealed an echogenic curvilinear structure with tense posterior acoustic shadowing seen extending from the periumbilical region to the left iliac fossa. Also, an ovoid cystic structure with multiple echogenic internal septation in the left adnexea, Pedunculated calcified uterine fibroid and mesenteric lymph node.

A diagnosis of Partial intestinal Obstruction $2^{\circ}$ post Op adhesion bands to rule out colonic tumour was made. She was admitted, Nasogastric tube and urethral catheter were passed, and intravenous fluid and analgesics were commenced. She was managed conservatively for 12 days with no improvement, hence was booked for exploratory laparotomy which was done with intraop findings of: Mid ileal mass measuring 16 x $6 \mathrm{~cm}$ comprising of intraluminal laparotomy sponge soaked in liquid faeces. Extensive adhesions surrounding the mass involving the jejunum, ileum, sigmoid colon. Uterus and ovary not visualized due to pelvic adhesions. Segmental ileal resection and anastomosis was done together with adhesiolysis.

Post Op recovery was essentially uneventful apart from superficial surgical site infection which was treated by wound dressing after which she was discharged on the $11^{\text {th }}$ day.

\section{Discussion}

Retained foreign body after surgery is an avoidable complication. It has serious professional and medicolegal consequences on the surgeon and the involved health facility [6]. Gossypiboma is the most common foreign material retained in the abdomen accounting for 70\% [7]. Gauze, mop, towels are used to control bleeding and clean the operating field during laparotomy. They are occasionally used to pack the paracolic gutters. Health workers practicing in rural health care facilities usually do not take necessary precautions to ensure that surgical instrument is removed from body cavities after surgery [8]. Female preponderance has been reported especially after gynaecological surgeries [9]. Some risk factors have been identified for this pathology: emergency operations, prolonged procedures, unplanned change in the course of a surgical procedure, involvement of more than one surgical team and patients with higher body mass index [10]. 
Transmural migration has been reported in the literature, though rare compared to abscess formation or formation of foreign body granuloma. Review of literature and medline search revealed 45 reported cases of transmural migration of surgical sponge following abdominal surgeries during the period of 2000-2010 [11]. This results from inflammation of the bowel wall with subsequent necrosis. The intestinal loop closes after complete migration of sponge leading to intestinal obstruction. The patient presents with abdominal pain, nausea, vomiting, anorexia, mass and weight loss [12]. Patil et al stated that the Fistulous tract is not usually seen, thus it is difficult to explain the course of the migration [13]. In our index patient, no fistulous tract was seen. The patient may present with features of intestinal obstruction as in index case, or sepsis, or enterocutaneous fistula or pseudotumor. This is one of the few reports of intraluminal migration of retained surgical sponge in Nigeria. Umoke et al [14]. in Gwagwalada Abuja reported a case of 31-year-old woman that presented with colicky abdominal pain of 10 months duration with associated vomiting. The patient had caesarean section 6 weeks prior to the onset of the symtoms. Clinical and radiological evaluation was not convincing and the patient was managed conservatively as a case of adhesive intestinal obstruction. The patient was eventually operated due to non-resolution of symptoms with intraop findings of intraluminal gauze in the small intestine. This case was similar to ours as the patient was initially managed as a case of adhesive intestinal obstruction. Terkimbi in Markudi reported a case of retained sanitary pad presenting with features of both colonic tumor and enterocutaneos fistula [8]. The index case also presented with features mimicking colonic tumor with a mass in the left iliac fossa

There are simple measures to prevent this complication: Keeping a thorough pack count, small sponges should not be used during laparotomy, tagging the packs with marker and thorough exploration of the surgical sites at the conclusion of the operation. New measures have been developed such as the use of radio-opaque sponge. Bar codes can be applied to all sponges, and with the use of bar code scanner, the sponges can be counted on the back table [15]. The condition is rare leading to low index of suspicion when the patient presents. This results in misdiagnosis and delay in management.

The diagnosis involves radiological imaging. Abdominal ultrasound scan findings include poorly defined echogenic area with intense posterior acoustic shadowing, or a well-circumscribed cystic mass containing internal mottled contents or nonspecific pattern simulating a complex mass [16].X-ray could identify radio-opaque swabs. Abdominal CT scan may show a rounded mass with dense central part and an enhancing wall. There is also whorl-like appearance with trapped air bubbles and cystic masses within the folded densities [13].

The management of this condition involves investigations, parenteral antibiotics, intravenous fluid and emergency laparotomy once the diagnosis is made like in our case. Enterotomy and extrusion of the mob is done. Currently, laparoscopic retrieval can also be done if the diagnosis is made early but this method is still infantile in our center [17].

\section{Conclusion}

Gozzypiboma is a frequent but avoidable medical error that occurs in medical practice and can be prevented by simple measures like keeping a pack count to always tagging the mop. This study is to sensitize surgeons on the possibility of this complication and the need to always adhere to the rules that prevent its occurrence.

\section{Compliance with ethical standards}

\section{Acknowledgments}

I wish to acknowledge the members of my team for always working together.

\section{Disclosure of conflict of interest}

There is no conflict of interest to declare.

\section{Statement of informed consent}

Informed consent was obtained from the individual whose case is being reported.

\section{References}

[1] Lauwers PR, Van Hee. Intraperitoneal gossypiboma: the need to count sponges. World J Surg. 2000; 24(5): 541527. 
[2] Tarik U, Gokhan DM, Sunnay YM. The medico-legal importance of gossypiboma. 4th Mediterranean Academy of Forensic Science Meeting, 14-18 October 2008. Antalya-Turkey. Abstract CD of Poster Presentations. 2009; 823.

[3] Sarda AK, Pandey D, Neogi S. Postoperative complications due to a retained surgical sponge. Singapore Med J. 2007; 48(8): 634-45.

[4] Al-Farah RU, Samia S. Intra-abdominal surgical gauze pseudotumor mimicking a dermoid cyst. Int J Pathol. 2012; 10: 85-7.

[5] Monika GA. A review of medicolegal consequences of gossypiboma. J Indian Acad Forensic Med. 2010; 32(4): 358-61.

[6] Stawicki SP, Evans DC, Cipolla J, Seamon MJ, Lukaszczyk JJ. Retained surgical foreign bodies: A comprehensive review of risks and preventive strategies. Scand J Surj. 2009; 98: 8-17.

[7] Bani-Hani KE, Gharaibeh KA. Retained surgical sponges(gossypiboma). Asian J Surg. 2005; 28(109-15).

[8] Terkimbi UB, Joseph IT. Retained post-operative foreign body of gynaecologic origin at ascending colon. Gynecol Obs Case Rep. 2016; 2(2).

[9] Kamal EB, Gharaibeh KA. Retained surgical sponges( Gossypiboma). Asian J Surg. 2005; 28(2): 109-15.

[10] Gawande AA, Studdert DM, Orav EJ, Brennan TA. Risk factors for retained instruments and sponges after surgery. N Engl J Med. 2003; 348: 229-35.

[11] Akbulut S, Arikanoglu Z, Yagmur R, Basbug M. Gossypibomas mimicking a splenic hydatid cyst and ileal tumor: Acase report and literature review. J Gastrointest Surg. 2011; 15: 2101-7.

[12] Dux M, Gantem M, Lubienski A. Retained surgical sponge with migration into the duodenum and persistent duodenal fistula. Eur Radiol. 2002; 12: 874-7.

[13] Patil KK, Patil SK, Gorad KP, Anuradha HP, Arora SS. Intraluminal migration of surgical sponge: Gossypiboma. Saudi J Gastroenterol. 2010; 16(3): 221-2.

[14] Umoke I, Stephen G, Henrietta U, Abubakar H. Spontaneous trans-visceral migration and intestinal obstruction due to textiloma: A case report. Int J Case Rep Images. 2019; 10: 1-6.

[15] Gibbs V, Coakley F, Reines H. Preventable errors in the operating room:Retained foreign bodies after surgerypart 1. Curr Probl Surg. 2007; 44: 261-337.

[16] Kumar GV, Ramani S, Mahajan A, Jain N, Sequeira R. Imaging of retained surgical items: Apictorial review including new innovations. Indian J Radiol Imaging. 2017; 27(3): 354-61.

[17] Uranus S, Schauer C, Pfeifer J, Dagcioglu A. Laparoscopic removal of a large laparotomy pad forgotten in situ. Laparosc Endosc. 1995; 5: 77-9. 